\title{
ITETRIS - A System for the Evaluation of Cooperative Traffic Management Solutions
}

\author{
D. Krajzewicz, R. Blokpoel, F. Cartolano, P. Cataldi, A. Gonzalez, O. Lazaro, J. Leguay, L. Lin, J. \\ Maneros, M. Rondinone
}

\begin{abstract}
V2X communication - communication between vehicles (V2V) and between vehicles and infrastructure (V2I) - promises new methods for traffic management by supplying new data and by opening new ways to inform drivers about the current situation on the roads. Currently, V2X cooperative systems are under development, forced by both the industry and by the European Commission which supports the development as a part of its Intelligent Car Initiative. Within this publication, "iTETRIS", a new system for simulating V2X-based traffic management applications is described which aims on high-quality simulations of large areas. This is achieved by coupling two well-known open source simulators. The sustainability of the project is guaranteed by making the whole also available as an open source tool.
\end{abstract}

\section{Introduction}

Mobility has had an increasing impact on worldwide economical and social development over the last decades, which is highlighted by the fact that up to $40 \%$ of World Bank loans have been used on transport projects [1]. However, current road traffic yields in high congestion and pollution and both will increase with the predicted increase of road traffic amount. One goal towards avoiding these problems and achieving sustainability in road traffic is to improve traffic management by intelligent information and communication technologies (ICT). Here, the wireless vehicular communications ("V2X communication" or "V2X" for short) technology gained a major interest in the recent years and became part of the EC's Intelligent Car Initiative [2]. Cooperative systems which use this technology will be able to assist the driver with additional knowledge gained through the dynamic exchange of messages between vehicles and between vehicles and infrastructure. A wide range of possible applications which use V2X are under development. They range from safety-related applications, such as collision warning or pre-crash warnings, up to applications which unfold their potentials in large areas, such as broadcasts of travel times and route guidance based on this information.

The European research activities with regard to cooperative systems started under the 6th Framework program with projects such as CVIS [3], Safespot [4] or Coopers [5]. To validate and better estimate the impact of cooperative systems, Field Operational Tests (FOTs) have been launched under the 7th Framework Programme. These FOTs will provide a needed feedback on the impact, performance, and predicted adoption of cooperative systems. Nevertheless, they are limited in size, time and the number of involved vehicles. For traffic management a view on large areas and different numbers of vehicles is needed to answer questions the new systems pose: What infrastructure is needed to support a certain traffic management strategy? Does a new strategy bring a benefit which justifies the costs? How does the system behave under different rates of vehicles equipped with V2X systems? And, above all: Will autonomous systems disturb centralized traffic management policies?

These questions can only be answered using simulations. Such simulations must be able to resemble a large area's road traffic, possible incidents, and the communication between a variable amount of vehicles equipped with $\mathrm{V} 2 \mathrm{X}$ devices, optionally including a variable amount of installed communication infrastructure. Additionally, the traffic management strategies that shall be evaluated must be modelled and incorporated into the simulation. Several approaches which allow such simulations exist and are well described [6-10]. Unfortunately, many of these simulations are not available to the public. Also, being developed in academic context, most of them lack a real support for potential users and get unsupported after the thesis in which context they were developed ends.

The European FP7 iTETRIS (Integrated Wireless and Traffic Platform for Real-Time Road Traffic Management Solutions, http://ict-itetris.eu/) project aims at implementing an open-source, integrated wireless and traffic simulation platform. It will allow testing and optimising V2V and V2I communications and cooperative traffic management strategies for large-scale scenarios. The sustainability will be guaranteed by a continuing its usage and dissemination after the project's end, as well as by using modern simulators and the possibility to exchange them by newer developments. 
Within this publication, the results of this project's first year are presented, starting with descriptions of real-world needs. Then, a set of strategies developed within the project are described. Afterwards, the simulation system architecture is presented, together with extensions of the used simulators the system consists of. We will close with a short outlook on the project's next steps.

\section{Investigation of a City's Problems}

In order to develop a tool which addresses real world problems, the first step was to analyze the needs of traffic management within a real city. The municipality of Bologna, one of iTETRIS project partners, evaluated typical traffic scenarios in the city. This analysis has taken into account different factors, such as urban structure, commuting, public transport offer, traffic control systems, etc. This preliminary work defined traffic flow parameters describing various large scale traffic test sites from the mobility point of view. In addition, specific congestion events were studied and classified, in order to collect a wide range of cases and to identify the possible causes for unexpected or recurring traffic jams. In the following, these results will be presented. At first, a description of the current mobility situation of the city of Bologna is given. Then, the generated traffic scenarios which describe the spatiotemporal shape of problematic zones as input to traffic simulations are presented.

\subsection{The Mobility in the Urban Area of Bologna}

The city of Bologna, located in the middle-north of Italy, constitutes an important crossroad for national mobility flows over north-south and east-west axis. Moreover, it has a very evocative historical centre that attracts many tourists during the year. The city has an important fair centre and one of the biggest universities in Italy (and the oldest continually operating one in Europe). The resident population in the city today is about 374,000 (the municipality area is $140.85 \mathrm{~km}^{2}$ ), while the metropolitan area, which extends to the neighboring towns, and more pertinent from the mobility's point of view, counts nearly 600,000 residents. There are about 180,000 local workers in the municipal area, while the daily city users are around 300,000. The result of this demographic structure is that traffic flows within the network are very heavy and include very short trips within the city, short distance trips among the main city and the towns belonging to the same province, medium distance trips taking place within the regional network of cities, and long distance through traffic with areas outside the region.

Like other medium-sized European cities, Bologna suffers because of the regularly high levels of urban traffic. In the last few years, traffic to and from the areas surrounding the city has increased. On the contrary, following new mobility management policies, there has been less traffic in the city itself. During a medium work-day approximately 2,000,000 movements are produced in Bologna; in particular, almost half of these movements is completely within the administrative city area, while the other half is equally distributed between city crossing trips and exchange ones with other cities. Private car use predominates in the exchange (approximately 70\%) and city crossing (approximately 90\%) movements. Considering only the inner movements, the private car use percentage is less than $40 \%$ because in urban travels people generally use public transportation, two wheeled vehicles, walk or bike.

\subsection{Recognized Bottlenecks and possible Solutions}

Three areas were chosen as being exemplary for a city's problematic zones. These three areas were used to set up scenarios, each defining the area they take place within, a description of the reasons of the problems occurring within this area, and possible strategies to solve these problems. Besides textual descriptions, the scenarios were modelled in Vissim or VISUM. These models, which include both the road network, and the traffic demand, could be transferred into a description usable by the traffic simulator SUMO which is used within the iTETRIS project for traffic modelling. In the following, the scenarios will be presented.

The first scenario describes an area affected by recurring events taking place at the city stadium, such as football matches or concerts. The chosen area also includes a hospital as well as a cemetery. The goal to achieve in this scenario is to manage the traffic in an area that offers few alternative routes, and where the stadium often generates a significant and extraordinary increase in traffic flow that affects adjacent areas of the traffic network. Possible solutions may include adapting traffic light timings, postponing driver departure times by offering information about current state in this part of the network, recommending routes around the affected area, or changing traffic regulation, e.g. by allowing private vehicles to use the bus lanes located in this area. 
Being an old city, Bologna is surrounded by an inner city ring-way, which draws a line between the Bologna-centre and the remaining urban city. The ring-way is equipped with plenty of traffic lights and detection loops. It can be covered clockwise or anti-clockwise. It is the only way to cross the city, because the area within it is a Limited Traffic Zone (LTZ) and only few vehicles are allowed to enter it. The only exception is "Via Irnerio", a street that represents a shortcut connection to the ring-way and has no restrictions in being passed. Via Irnerio has a strong public transportation offer and a big open market on weekends which attracts a lot of vehicles and pedestrians. Here, the goals of traffic management are to reduce congestions on the roads which result from the high amount of traffic that crosses the city, from broken induction loops that make the installed control not to work, or from strikes or other events involving a large number of pedestrians who block the street. Solutions proposed for solving these problems are to adapt traffic light timings to the current situation, to detect and replace malfunctioning induction loop detectors by other traffic surveillance methods, and to disseminate routing information allowing drivers to pass the area more quickly.

The third investigated area is the highway located to the north of the city. An "Orbital" road runs beside the highway and, since 4 out of the 5 highway exits are connected directly to the Orbital, the Orbital is often chosen by drivers as an alternative route to the highway. As a result, the Orbital is often congested. The traffic management goals are to reduce the travel times and solve congestions of the Orbital. The proposed solution is to establish a routing support for drivers proposing them a certain exit depending on their destinations and the current traffic situation on the Orbital and on the highway, in terms of travel times.

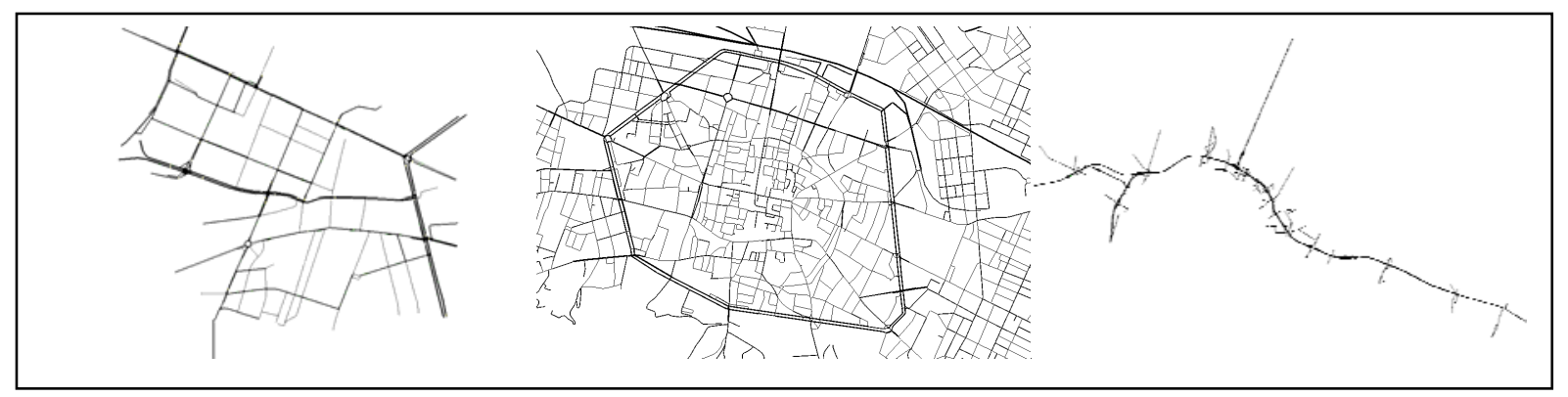

Fig. 1. iTETRIS areas; from left to right: city stadium area, inner city ring, highway area

\section{V2X Management Strategies}

Traffic management, in general, means optimizing the supply of road infrastructure for a given traffic demand. This process is based on the knowledge about the situation on the considered road network that is mainly described by the number of vehicles and their velocities. Additionally, traffic management involves informing road users about accidents, construction work, road closures or rerouting advices etc.

Conventional traffic surveillance systems, such as induction loops, are relatively expensive (some thousands Euros each). This usually disallows an area-wide coverage of the investigated road network. Furthermore, induction loops have a limited operational reliability over the time. Besides induction loops, other surveillance systems exist, such as cameras which detect and track vehicles or GSM-based level-of-service determination. However, it should be stated that such systems are not yet used on a large scale. All these techniques measure traffic at discrete points or along certain routes, only. Network-wide considerations of the actual traffic situation are not possible using nowadays methods. In conventional traffic management, road users are either informed by radio broadcasts of traffic reports or by variable message signs (VMS). Traffic reports are updated seldom and therefore tend to be outdated in a considerable number of cases. VMS are only situated on larger roads and their number is limited. For these reasons, only a part of road users can be provided with correct traffic information. Newer possibilities for supplying information are navigation devices of the third generation with an updated traffic situation.

Cooperative traffic management tries to close the gap of lacking data about the state of the considered road network by exchanging information between vehicles and between vehicles and infrastructure. In dependence to the equipment rate of vehicles and the number of installed road side units ("RSUs" or "ITS Roadside Stations") a high coverage, ranging up to a complete knowledge about all vehicles (cars, busses) on the road network is theoretically achievable. This knowledge offers new ways of managing traffic efficiently. I2V and V2V communication also promises to offer new possibilities to disseminate information about a road network's current and predicted state, reaching all equipped vehicles. Gathered or generated information can be both presented to a driver directly, or being processed by in-vehicle devices, generating only relevant information. 
Within iTETRIS, traffic management methods based on vehicular communication were developed and formally described. They were mainly derived from the traffic management proposals from the work on evaluation Bologna's traffic, presented in the last section. The results were descriptions of the 15 methods which can be combined into complex traffic management strategies. These methods can be grouped into four topics, as presented in the following:

- Surveillance: “Distributed traffic jam ahead detection”, “Travel time estimation”, “Identification of malfunctioning loop detectors”, "Induction loop replacement”, “(Decentralized) Floating car data”

- Navigation and Guidance: "Bus lane management”, "Limited access warning”, "Request-based personalized navigation”, "Postpone departure time for road network balancing”, "Decentralized route recommendation based on travel time estimation", "Routing in traffic light controlled network"

- Traffic Lights: “Emergency vehicle prioritization”, “Traffic light adaptation by traffic management centre”

Assistance Systems: "Regulatory and contextual speed limit information”, "Event based traffic condition notification”

Each method was described in a high detail, including a) a textual descriptions, b) information about actors, used messages, communication mode and required network, expiry time, and transmission frequency, c) a diagram showing the data processing within the involved actors if the method would be implemented in reality, d) a sequence diagram showing the data flow for simulating the method, e) a diagram and descriptions of the structures needed for simulating the method, and f) the derived requirements to the simulation system and to the involved simulators.

\section{Simulation System}

The developed strategies will be evaluated using a simulation system which is the major result of the iTETRIS project. The simulation system couples two well-known simulators, ns-3 [11] for networking simulation, and SUMO [12] for traffic simulation. Both simulation applications are available as open source. Furthermore, instances of the strategies to evaluate are run as external applications. In the following, the design of the overall simulation system is presented, first, including the reasons for choosing it. Then, extensions to the used simulators implemented within the iTETRIS project are outlined.

\subsection{System Architecture}

Different approaches for simultaneous, coupled simulation of communication and traffic were used in the past: a) "Integrated Simulation" where the simulation is performed using a single program which includes modules for the communication, the application, and the traffic simulation $([9,10])$, b) "Direct Simulator Coupling" where two simulators (SUMO and ns-2 for example) are directly coupled, for example using a socket connection and the application simulation is built into one of the simulators, usually into the communication simulation ([7]), c) "ThreeBlocks Architecture" where two simulators are coupled using a control module "in-between" which is responsible for the synchronization, translation of messages, etc., and mostly also contains the application logic ([6]), and d) usage of a "Standardized Distributed Simulation Architecture" where all simulations, including the application simulation are connected to a communication middleware which does not hold any simulation itself, but is responsible for realizing communication between the underlying simulators and overall synchronization only ([8]).

All architectures are often realised using already existing applications for traffic and communication simulation and connecting those using different methods. However, connecting simulations is not straight-forward. Often, the simulations do not offer the possibility to be accessed or externally controlled. Furthermore, the given interfaces are not standardised and special access methods must be implemented, often including the usage of proprietary and not portable libraries. Additionally, a mapping between different representations of simulated instances, for example between the different names of a vehicle must be established.

In order to decide on an architecture that fits to iTETRIS' needs, the wanted system's features were put against the different approaches. The following briefly given thoughts yield to a decision: 
Interoperability and Sustainability: Both used simulators should be exchangeable by new developments; this forbids using an integrated approach where both simulators would be merged into a single application.

- Performance and Scalability: If only one computer is used for simulation, an integrated approach promises a high execution speed by avoiding the need to exchange and translate information between different simulators. Nonetheless, solutions coupling simulators allow an easier parallelisation.

Extensibility and Maintainability: Both integrated approaches, and direct coupling of simulators would yield in large and complicated software structures which contain parts from "both worlds" - the road traffic simulation and the communication simulation.

Implementation Effort and Testing: Keeping the involved simulations separately, allows separate developing and testing. The implementation effort and the software's are additionally raised by keeping the focus on a certain problem, e.g. traffic simulation.

The final decision was to couple both involved simulators and the simulated applications to a middleware instance called iCS (iTETRIS Control System) using socket connections, as shown in Figure 2. The middleware instance is responsible for translating information between the simulator and application instances and for synchronizing them. In addition, it contains structures enriching and storing information obtained from both simulators making the whole simulation system compliant with the COMeSafety-architecture [13].

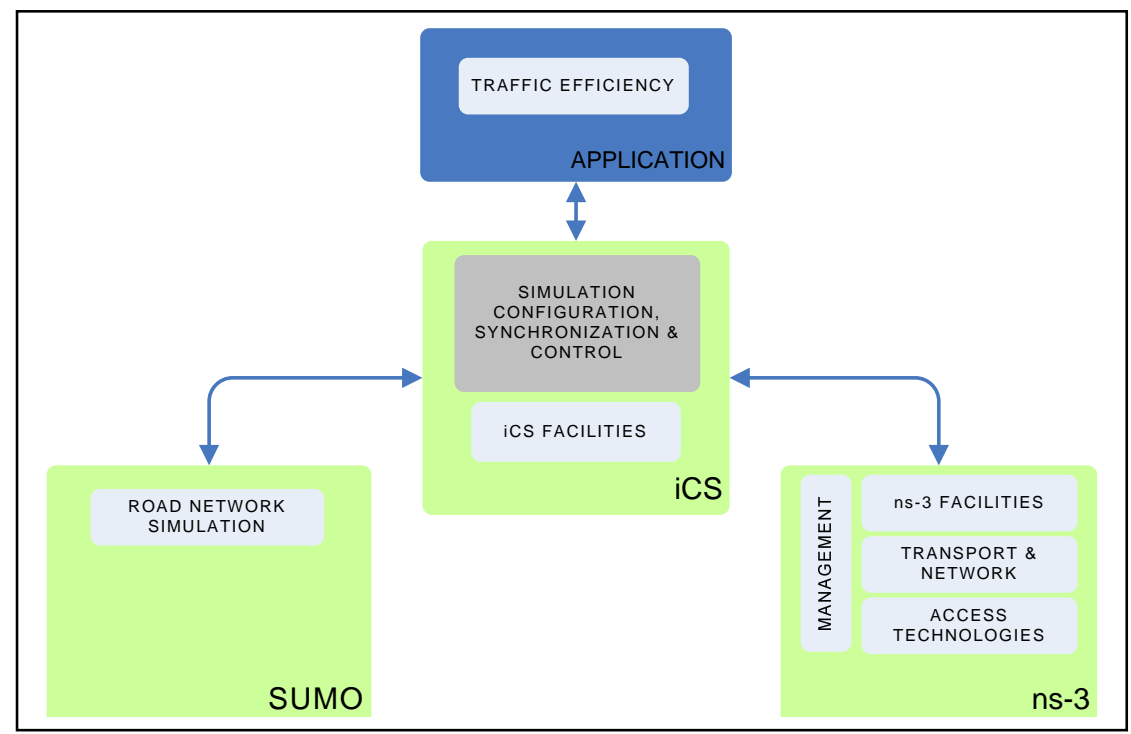

Fig. 2. Chosen Simulation System Architecture

\subsection{Extensions to the involved Simulators}

Both used simulators, ns-3, and SUMO had to be extended for being suitable to model the questions addressed by the iTETRIS project. ns-3 had no support for V2X wireless communication simulation and had therefore to be extended. A new module was created to emulate radio transmissions over ITS G5A, the European version of the IEEE 802.11p communication technology (also known as WAVE), being in turn an amendment of the 802.11 standard allowing communications in vehicular environments. A similar work was made for the creation or adaptation of specific modules for the other radio technologies considered in the project: UMTS, WiMAX and DVB-H. Moreover, in order to be compliant with the COMeSafety ITS Communication Architecture, new modules and functionalities are being added to represent all the layers of the communication stack needed for wireless communication in an ITS scenario (see figure 2). Additionally, a communication module named inCI (iCS to ns-3 connection interface) for coupling ns-3 to the iTETRIS Control System was implemented.

The communication module of SUMO was extended in order to support the simulation of traffic management algorithms, by allowing to access and manipulate simulated traffic lights, induction loops, and vehicles. Because a 
very strong attention is put on developing strategies which show the ecological benefits of strategies based on V2X communication, SUMO was extended in order to be able to compute vehicular pollutant - $\mathrm{CO}, \mathrm{CO}_{2}, \mathrm{PM}_{\mathbf{x}}, \mathrm{HC} \mathrm{NO}_{\mathbf{x}}$ - and noise emissions as well as fuel consumption.

\section{Conclusions and Outlook}

The iTETRIS project will deliver a system for simulating large-scale traffic management applications and strategies which use V2V and V2X communication in high quality. The sustainability of the resulting simulation system is guaranteed by keeping it open for new simulation tools and by releasing it under an open source licence. The resulting application offers a missing tool for traffic engineers allowing them to measure the benefit of new cooperative solutions before implementing them in reality.

\section{References}

[1] Department for Transport, UK Government, Transport and the economy: full report (SACTRA)

[2] European Commission, Intelligent Car Initiative Web Portal, http://ec.europa.eu/information_society/activities/intelligentcar/index_de.htm, last visited on 10.02 .2010

[3] CVIS project home page, http://www.cvisproject.org/, last visited on 10.02.2010

[4] Safespot project home page, http://www.safespot-eu.org/, last visited on 10.02.2010

[5] Coopers project home page, http://www.coopers-ip.eu/, last visited on 10.02.2010

[6] Piórkowski M, Raya $M$ et al, TraNS: Realistic Joint Traffic and Network Simulator for VANETs, SIGMOBILE Mob. Comput. Commun. Rev., Vol 12, No 1, ISSN 1559-1662. Pages 31—33, 2008, doi: http://doi.acm.org/10.1145/1374512.1374522

[7] Wegener A, Hellbrück M, et al, VANET Simulation Environment with Feedback Loop and its Application to Traffic Light Assistance, Proceedings of the 3rd IEEE Workshop on Automotive Networking and Applications, New Orleans, LA, USA, 2008

[8] Queck T, Schuenemann B, Radusch I, Runtime Infrastructure for Simulating Vehicle-2-X Communication Scenarios, Proceedings of the Fifth ACM international Workshop on Vehicular inter-Networking, San Francisco, California, USA, September 15, VANET '08, ACM, pp. 78, New York, NY, USA, ISBN: 978-1-60558-191-0, 2008

[9] Kerner B, Klenov S, and Brakemeier A, "Testbed for wireless vehicle communication: A simulation approach based on three-phase traffic theory," Proceedings of the IEEE Intelligent Vehicles Symposium (IV'08), pp. 180-185, 2008

[10] Krajzewicz D, Teta Boyom D, Wagner P, Untersuchungen der Performanz einer auf C2CKommunikation basierenden, autonomen Routenwahl bei Stauszenarien, Heureka '08, 2008-03-05 - 2008-03-06, 2007

[11] ns-3 home page, http://www.nsnam.org/, last visited on 10.02.2010

[12] SUMO home page, http://sumo.sf.net/, last visited on 10.02.2010

[13] COMeSafety Project, "European ITS Communication Arctitecture, Overall Framework, Proof of Concept Implementation" version 3.0, February 2010

Daniel Krajzewicz

Institute of Transportation Systems, German Aerospace Center

Rutherfordstr. 2

Berlin 


\section{Germany}

E-mail: Daniel.Krajzewicz@dlr.de

Robbin J. Blokpoel

Peek Traffic bv

Basicweg 16

Postbus 2542

3800 GB Amersfoort

Netherlands

E-mail: Robbin.Blokpoel@peektraffic.nl

Fabio Cartolano

Comune di Bologna

Dipartimento Qualità della Città - Settore Mobilità - U.O. Sviluppo Sistemi Telematici

P.zza Liber Paradisus, 10

40129 Bologna

Italy

E-mail: Fabio.Cartolano@comune.bologna.it

Pasquale Cataldi

Mobile Communication Department, EURECOM

2229 route des crêtes

F-06560 Sophia-Antipolis cedex

France

E-mail: pasquale.cataldi@eurecom.fr

Ainara Gonzalez

Innovalia Association

Rodriguez Arias, 6

48008 Bilbao

Spain

E-mail: aigonzalez@innovalia.org

Oscar Lazaro

Innovalia Association

Rodriguez Arias, 6

48008 Bilbao

Spain

E-mail: olazaro@innovalia.org

Jérémie Leguay

Thales Communication France

160, boulevard de Valmy

92706 Colombes Cedex

France

E-mail: jeremie.leguay@fr.thalesgroup.com

Lan Lin

Hitachi Europe Ltd.

1503 Route des Dolines

06560 Valbonne - Sophia Antipolis

France

E-mail: lan.lin@hitachi-eu.com

Julen Maneros 
CBT Comunicación \& Multimedia

Carretera Asua, 6

48930 Getxo

E-mail: jmaneros@cbt.es

Michele Rondinone

Uwicore Laboratory

University Miguel Hernández of Elche

Avda de la Universidad, s/n

03202, Elche (Alicante)

Spain

E-mail: mrondinone@umh.es

Keywords: traffic management, V2V communication, simulation, traffic management strategies 